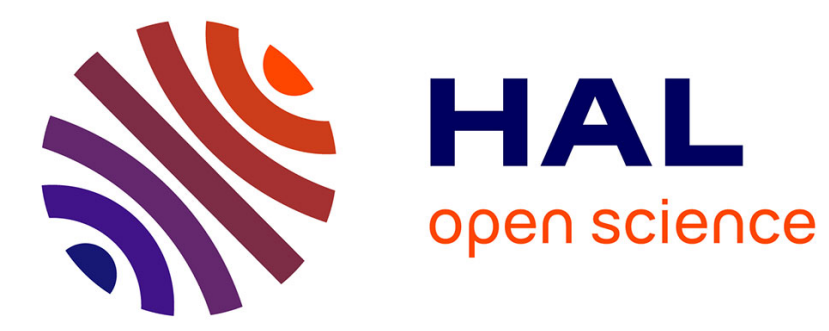

\title{
Stochastic Battery Operations using Deep Neural Networks
}

\author{
Yize Chen, Md Umar Hashmi, Deepjyoti Deka, Michael Chertkov
}

\section{To cite this version:}

Yize Chen, Md Umar Hashmi, Deepjyoti Deka, Michael Chertkov. Stochastic Battery Operations using Deep Neural Networks. IEEE ISGT NA 2019 - Innovative Smart Grid Technologies Conference, Feb 2019, Washington, United States. hal-02070232

\section{HAL Id: hal-02070232 https://hal.science/hal-02070232}

Submitted on 17 Mar 2019

HAL is a multi-disciplinary open access archive for the deposit and dissemination of scientific research documents, whether they are published or not. The documents may come from teaching and research institutions in France or abroad, or from public or private research centers.
L'archive ouverte pluridisciplinaire HAL, est destinée au dépôt et à la diffusion de documents scientifiques de niveau recherche, publiés ou non, émanant des établissements d'enseignement et de recherche français ou étrangers, des laboratoires publics ou privés. 


\section{Stochastic Battery Operations using Deep Neural Networks}

\author{
Yize Chen \\ Dept. of Electrical Engineering \\ University of Washington, Seattle, WA \\ yizechen@uw.edu
}

\author{
Md Umar Hashmi \\ INRIA, DI ENS \\ PSL University, Paris, France \\ md-umar.hashmi@inria.fr
}

\author{
Deepjyoti Deka, Michael Chertkov \\ Theory Division \\ Los Alamos National Lab, Los Alamos, NM \\ (deepjyoti, chertkov)@lanl.gov
}

\begin{abstract}
In this paper, we introduce a scenario-based optimal control framework to account for the forecast uncertainty in battery arbitrage problems. Due to the uncertainty of prices and variations of forecast errors, it is challenging for battery operators to design profitable strategies in electricity markets. Without any explicit assumption or model for electricity price forecasts' uncertainties, we generate future price scenarios via a data-driven, learning-based approach. By aiding the predictive control with such scenarios representing possible realizations of future markets, our proposed real-time controller seeks the optimal charge/discharge levels to maximize profits. Simulation results on a case-study of California-based batteries and prices show that our proposed method can bring higher profits for different battery parameters.

Index Terms-Battery energy storage, generative model, machine learning, power system economics, scenario forecasts
\end{abstract}

\section{INTRODUCTION}

In recent years, energy storage has become an integral part of modern grid operations. On the one hand, higher penetration of uncertain renewables, stochastic net demand, and varying user electricity consumption call for storage systems to provide resiliency and reliability; on the other hand, the decreasing costs of storage and the variability in real-time electricity prices have brought potential economic benefits to battery owners. Energy storage arbitrage refers to such revenue opportunities, where the battery owner charges the battery when prices are low and discharges it when prices are high [1], [2].

Several pioneering research have studied the economic benefits brought by operating energy storage [3]. However, these works mainly assume that the battery operator possesses perfect knowledge of future electricity prices. In reality, such assumption can be too restrictive making it non-trivial to design a feasible and efficient strategy for battery owners to maximize their profits in real time [4]. The main obstacle here arise due to imperfections in the uncertainty modeling of forecast prices. Electricity price can be forecast based on historical observations and market signals, yet any off-theshelf forecasting methods would bring prediction errors into subsequent battery control and arbitrage decisions. Battery owners need to design a strategy that maximizes accumulated

The authors acknowledge the support from the U.S. Department of Energy through the Grid Modernization Lab Consortium, and the Center for Non Linear Studies at Los Alamos National Laboratory.

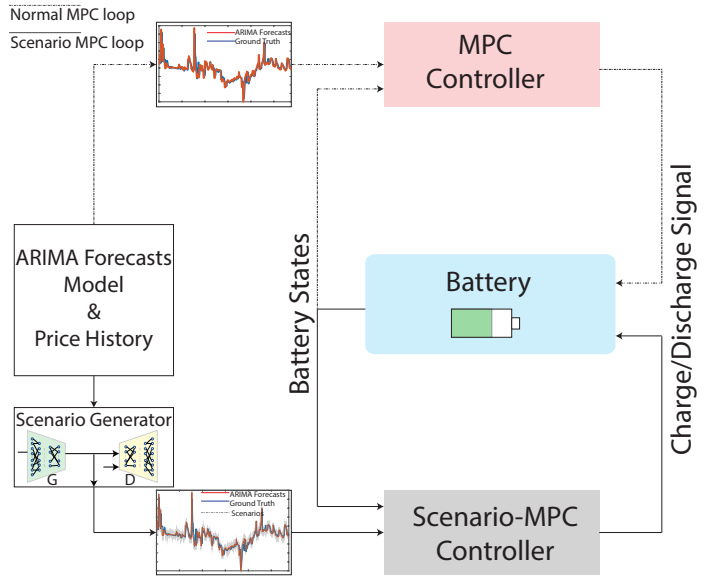

Fig. 1. Schematic plot for our scenario-based battery controller, with a comparison to normal MPC controller using point forecasts.

profits for the overall operation period where designed charging/discharging actions are coupled in time due to finite size of inventory or capacity, inaccurate forecasts can lead to suboptimal and hence less profitable sequential decisions.

To deal with such forecasting uncertainties, researchers have tried to design algorithms that are more robust to predictions errors, such as ones based on model predictive control (MPC) and dynamic programming (DP). The authors in [5], [6] maximize the expected value in presence of uncertain parameters, though the solutions are very sensitive to the accuracy of given point forecast algorithms. The authors in [7] propose multi-stage forecast for receding horizon control to mitigate the effects of forecasting errors, yet it is hard to quantify the uncertainty explicitly. In [8], the authors use a data-driven reinforcement learning algorithm to maximize the arbitrage, yet both battery charging level and charge/discharge actions are discrete and simplified. From another perspective, other researchers tried to handle the uncertainties explicitly via robust optimization [9], chance-constraints [10] or scenariobased optimization [11]. The scenario-based approach often avoids the conservative solutions achieved by robust optimization. It uses independent, identically distributed future realizations (scenarios) to expressively represent the uncertainties. Moreover, with an appropriate choice of the number of the scenarios, the solution of the scenario-based problem can be made a feasible solution of the corresponding chance- 
constrained problem [11]. Scenario approach can also ease the subsequent control and optimization problem with convex and linear formulations that are easy to scale. In [12], [13], authors use scenario-based maximization for selecting control decisions for arbitrage problem. However, their scenario generation process is model-based, which still holds assumptions on the distribution on forecasting errors.

In this work, we focus on incorporating the uncertainties of electricity prices, and tackle the battery temporal arbitrage problem by combining the power of predictive control with a model-free scenario generation algorithm. By formulating the arbitrage problem as a scenario-based model predictive control (SMPC) problem [14], we derive a computationally efficient algorithm for finding optimal control inputs over a finite horizon. As shown in Fig. 1, compared to classical MPC control algorithm, we design the scenario-MPC controller, and derive the optimal charge/discharge actions with respect to certain number of future price scenarios. Moreover, our method can be applied on top of any forecast method such as autoregressive integrated moving average (ARIMA) or learning method, and we make no assumptions on the distribution or the temporal correlations of forecasting prices. Instead we generate a group of forecasting scenarios by adopting the datadriven, learning-based scenario generation method proposed in our prior work [15], [16]. In summary, there are two key contributions of this paper:

- Efficient online arbitrage with model-free scenarios: We extend a MPC algorithm for battery arbitrage proposed in [2],[17] with model-free scenarios generated by deep learning generative model. The benefit of our approach is that it is able to learn the distribution and temporal correlation of future forecasts, and is amenable to changing forecasts on the fly by conditioning it on the past.

- Practicability of battery arbitrage: we conduct extensive simulations on real price data to validate our proposed algorithm on battery arbitrage with varying ramp rates. Comparisons to MPC using point forecasts or day-ahead price illustrate superiority of proposed method.

\section{Battery Modeling ANd Problem Description}

In this section, we start with the modeling of a battery storage, which includes its charging/discharging behaviors as well as its participation in real-time market. We then formulate the deterministic version of battery arbitrage problem, which serves as the preliminary model for our proposed scenariobased control algorithm. The optimal arbitrage problem considered in this paper is a special case of [17] where the price of electricity for buying and selling at any time instant are the same [2].

\section{A. Battery Model}

We focus on an energy storage operating over a finite horizon $\mathscr{T}=\{1, \ldots, T\}$. The duration of each discrete step $t \in\{1, \ldots, T\}$ is denoted as $h$. The efficiency of charging and discharging of battery is denoted by $\eta_{c h} \in(0,1]$ and $\eta_{\text {dis }} \in(0,1]$, respectively. We denote the change in the energy level (e.g., charge/discharge action) of the battery at $t^{t h}$ instant by $u_{t}=h \delta_{t}$, where $\delta_{t}$ denoted the storage ramp rate at the $t^{t h}$ instant such that $\delta_{t} \in\left[\delta_{\min }, \delta_{\max }\right], \forall t$ and $\delta_{\min } \leq 0$ and $\delta_{\max } \geq 0$ are the minimum and maximum ramp rates $(\mathrm{kW}) ; \delta_{t}>0$ implies charging and $\delta_{t}<0$ implies discharging. At time $t$, energy level $b_{t+1}$ is changed from $b_{t}$ through $b_{t+1}=b_{t}+\frac{\left[u_{t}\right]^{+}}{\eta_{c h}}-\eta_{d i s}\left[u_{t}\right]^{-}$, where $[x]^{+}=\max (0, a)$ and $[x]^{-}=-\min (0, a)$. The rated battery capacity is denoted as $B_{\text {rated }}$. The state-of-charge of the battery is denoted as $S o C_{t}=\frac{b_{t}}{B_{\text {rated }}}$. The SoC is bounded by $S o C_{i} \in\left[S o C_{\min }, S o C_{\max }\right]$, in order to avoid overcharging or over-discharging. Therefore, the battery should operate within $b_{\min }=S o C_{\min } B_{\text {rated }}$ and $b_{\max }=S o C_{\max } B_{\text {rated }}$.

\section{B. Deterministic MPC Formulation}

We now formulate the finite horizon optimal control problem for battery arbitrage problem. We follow the MPC principle to design our control algorithm, where for a fixed horizon $\mathscr{T}$, we find $\mathbf{u} \in \mathbb{R}^{T}$ at $t=1$, and we implement $u_{1}$ and proceed to the next time-step.

The electricity price at instance $t$ is denoted as $p_{t}$. Since future prices $p_{t}, t=1, \ldots, T$ are unknown, we use the price forecasts information $\hat{p}_{t}$ in our deterministic version of MPC. Then the battery arbitrage problem for $\mathscr{T}$ can be formulated as below:

$$
\begin{array}{ll}
\min _{u_{t}} & \sum_{t=1}^{T} \hat{p}_{t}\left(\left[u_{t}\right]^{+} / \eta_{c h}-\eta_{d i s}\left[u_{t}\right]^{-}\right) \\
\text {s.t. } & b_{t+1}=b_{t}+\left[u_{t}\right]^{+} / \eta_{c h}-\eta_{d i s}\left[u_{t}\right]^{-} \\
& \delta_{\min } h \leq u_{t} \leq \delta_{\max } h \\
& b_{\min } \leq b_{t} \leq b_{\max }
\end{array}
$$

where Eq.(1b) is the dynamics of the battery, while Eq.(1c) and Eq.(1d) constrain the charge/discharge level and battery level respectively. To solve Eq. (1) efficiently, we define the electricity price for battery in this case as

$$
\hat{p}_{t}^{b a t}= \begin{cases}\hat{p}_{t}^{c h}=\hat{p}_{t} \frac{1}{\eta_{c h}}, & \text { if } u_{t} \geq 0, \\ \hat{p}_{t}^{d i s}=\hat{p}_{t} \eta_{d i s}, & \text { otherwise }\end{cases}
$$

We also denote $U_{\min }=\delta_{\min } h, U_{\max }=\delta_{\max } h$. Then the arbitrage problem defined in Eq. (1) can be reformulated as

$$
\begin{array}{ll}
\min _{u_{t}} & \sum_{t=1}^{T} \hat{p}_{t}^{b a t} u_{t} \\
\text { s.t. } & \text { Eq.(1b), Eq.(1c), Eq.(1d) }
\end{array}
$$

Using the convexity of the objective and constraints, an algorithm for optimal arbitrage was proposed in our prior work [2],[18] based on optimal Lagrange multipliers [19]. This algorithm highlights that optimal control decisions are independent of past or future values of prices beyond a 'sub-horizon', where a sub-horizon is a portion of the entire time horizon where the shadow price of transactions remains constant.

In some cases with high penetration of renewables, there may exist negative prices. Note that under such cases, the 


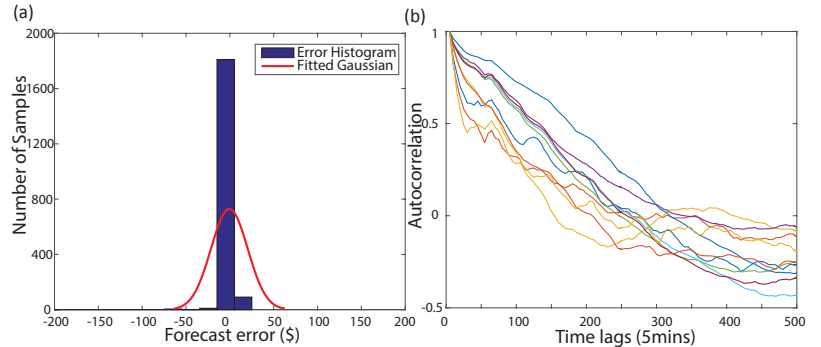

Fig. 2. (a). The error distribution on one-week's ARIMA forecasted future price; (b). Autocorrelation plot for 10 randomly selected daily electricity price samples.

cost function of the optimization is no longer convex. Here we make a realistic assumption that negative prices are rare, and assume the optimal action for the consumer under negative prices is to charge. For instance in Germany and in California the negative prices comprised of $2.6 \%$ of total hourly prices for the year $2017^{12}$. Under this condition the threshold-based structure, used for selecting optimal control decisions in prior work [2], is modified to Eq. 4 in Remark 1.

Remark 1: The optimal control decision $u_{t}^{*}$ for the $t^{\text {th }}$ instant minimizes the function $C_{t}^{\text {storage }}(u)-\mu_{t}^{*} x$ for $u \in\left[U_{\min }, U_{\max }\right]$. The optimal decision $u_{t}^{*}(\mu)$ is given as

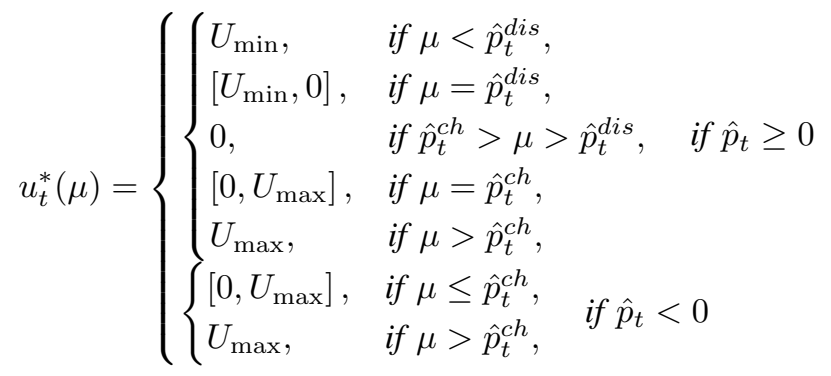

where $C_{t}^{\text {storage }}(u)=u_{t} \hat{p}_{t}$ and $\mu$ the accumulated Lagrange multiplier represents the shadow price of the transaction.

Note that for $\mu=\hat{p}_{t}^{c h}$ or $\mu=\hat{p}_{t}^{\text {dis }}, u_{t}^{*}(\mu)$ takes an envelope of values. For any other value of $\mu$ it is a singleton set. In order to find optimal decisions among an envelope of possible solutions based on the price variations, a backward Step algorithm is used as described in detail in our prior work [2]. We refer to it as OptimalArbitrage algorithm in this work.

\section{SCENARIO-BASED MPC}

Though the OptimalArbitrage algorithm could provide resiliency to forecast uncertainties by iteratively solving the MPC, it still heavily relies on the performance of forecasts, and the point forecast errors can still lead to unsatisfactory charging/discharging actions. As shown in Fig. 2a, the forecast errors are distributed in a very large interval of $[\$-200, \$ 200]$ dollars per MW, while the varying autocorrelation (Fig. 2b) pertaining to price data also increases the forecasting difficulty. To alleviate the effect of such uncertainties on forecasts, we

\footnotetext{
${ }^{1}$ https://tinyurl.com/y9xq5gul

${ }^{2} \mathrm{https}: / /$ tinyurl.com/y $87 \mathrm{t} 578 \mathrm{~b}$
}

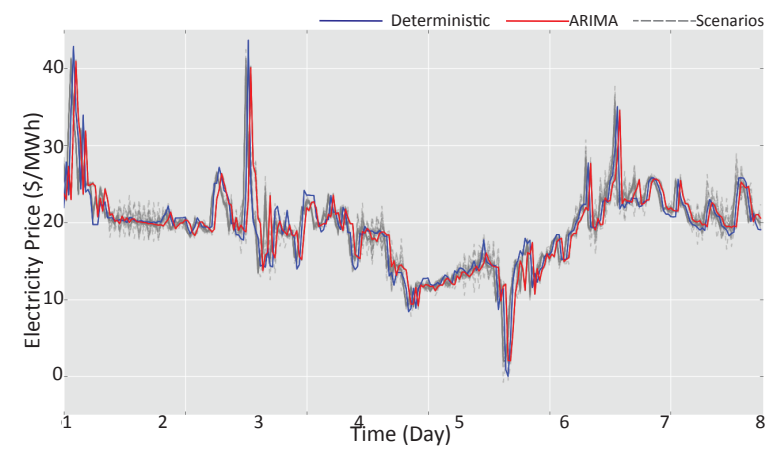

Fig. 3. Visualization of ground-truth (deterministic), ARIMA forecasts and generated scenarios of one-week electricity price from CAISO. Scenarios generated by our method can cover the mismatch between forecasts and real values.

make use of historical price observations and forecasts records, and train a data-driven learning model to generate scenarios for future prices. We then cast the battery arbitrage problem as a scenario-based MPC based on Eq. (3).

\section{A. Scenario-Based MPC}

Scenario approach has been introduced into optimization problems to replace the uncertainty set of variables by a finite number of scenarios. In [11], [20] different approaches have been proposed to formulate the uncertainty set as scenarios either in objectives or constraints. Suppose we generate a set of $K$ i.i.d scenarios $\hat{\mathbf{p}}_{\mathbf{1}}, \ldots, \hat{\mathbf{p}}_{\mathbf{K}}$ where $\hat{\mathbf{p}}_{\mathbf{k}}:=\left[p_{1, k}, \ldots, p_{T, k}\right]$. We follow the setup in [14] to adapt Eq.(3) to the scenario-based MPC (SMPC):

$$
\begin{array}{ll}
\min _{u_{t}} & \sum_{k=1}^{K} \sum_{t=1}^{T} \hat{p}_{t, k}^{b a t} u_{t} \\
\text { s.t. } & E q .(1 \mathrm{~b}), E q \cdot(1 \mathrm{c}), E q .(1 \mathrm{~d}), \forall t
\end{array}
$$

where (5a) incorporates $K$ scenarios (or equivalently, price state trajectories) into the main objective. The problem essentially finds the same $\mathbf{u}^{*}$ for $K$ different scenarios' to minimize the overall arbitrage costs, which can be solved efficiently via OptimalArbitrage proposed in [2], [17].

\section{B. Model-Free Scenario Generation}

Previous methods usually hold an assumption on the forecasting error (e.g., multi-dimensional Gaussian distribution), and generate scenarios via copula model or Lloyd-Max algorithm based on provided point forecasts [12], [13]. Here we take an alternative data-driven, model-free approach to overcome all assumptions. To generate a group of scenarios $\hat{\mathbf{p}}_{\mathbf{1}}, \ldots, \hat{\mathbf{p}}_{\mathrm{K}}$, we first collect two data-sets on historical records: (a). the historical electricity price $P$ and (b). the historical forecasts $\hat{P}^{3}$. We then construct the forecast error data-sets $X:=\{\hat{\mathbf{p}}-\mathbf{p}\}, \forall \mathbf{p} \in P, \hat{\mathbf{p}} \in \hat{P}$. We are interested in generating scenarios from the distribution $\mathbb{P}_{X}$, which is hard to model. The generative adversarial networks (GANs)[21] provides us an efficient deep neural network model to transform samples

\footnotetext{
${ }^{3}$ Our approach works for any forecast method, and we take ARIMA in all of our simulations.
} 

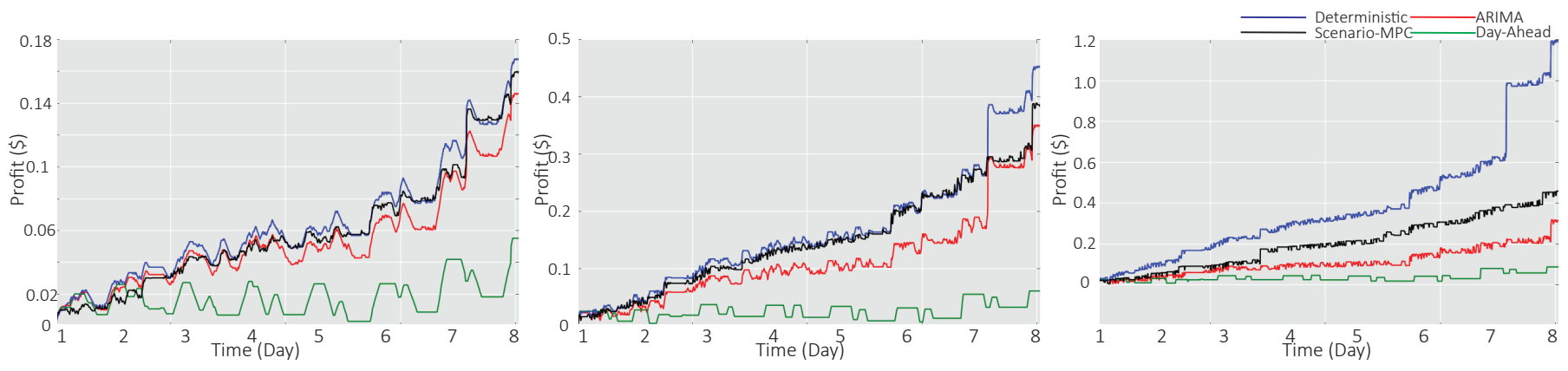

Fig. 4. Case studies on one-week profits of 3 batteries with varying ramp rate. By incorporating scenarios to account for the forecasting uncertainties, scenario-MPC outperforms control algorithms using pure ARIMA or day-ahead forecasts information.

from $\mathbb{P}_{Z}$ to samples in $\mathbb{P}_{X}$, where $\mathbb{P}_{Z}$ is a pre-defined, easyto-sample distribution (e.g., Gaussian). Following the steps described in [15], we train two neural networks: the generator $G(z)$ and discriminator $D(x)$ iteratively with following loss:

$$
\min _{G} \max _{D} \mathbb{E}_{X}[D(X)]-\mathbb{E}_{Z}[D(G(Z))]
$$

until generated samples from $G(Z)$ are statistically as real as samples coming from the ground truth error dataset $X$. We then try to find a group of $\hat{\mathbf{x}}_{\mathbf{k}}=G\left(z_{k}\right), k=1, \ldots, K$ based on the optimization step described in [16] to represent all the possible errors of the price forecasts conditioned on historical error observations. Finally we get the forecasting scenarios by combining point forecasts $\hat{\mathbf{p}}$ with $\hat{\mathbf{x}}_{\mathbf{k}}$. We refer these scenario generation steps as ModelFreeScenarios algorithm. In Fig. 3 we show one-week's price scenario along with the ground truth and ARIMA forecasts, where our scenarios could cover the uncertainties in ARIMA prediction.

\section{Online algorithm}

We can now bridge the machine learning scenario generation algorithm ModelFreeScenarios with the optimal control algorithm OptimalArbitrage into the SMPC formulation described in Eq.(5). We term the entire process as ScenarioMPC algorithm for real-time battery arbitrage.

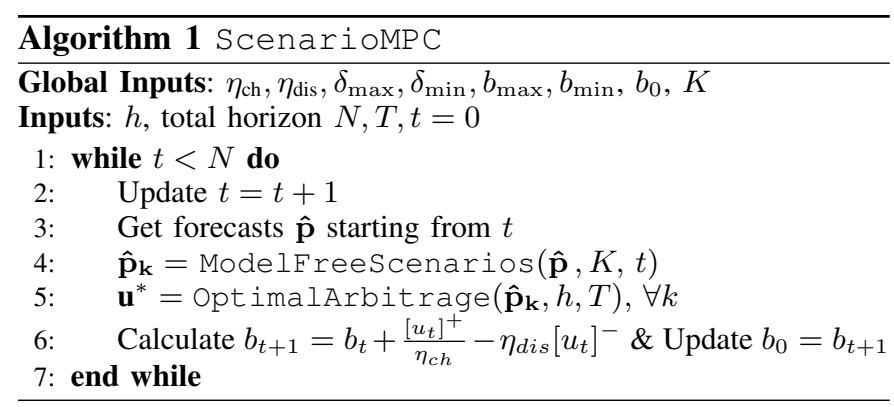

\section{NumericAl CASE STUdY}

\section{A. Simulation Setup}

We validate the performance of our algorithm on three battery model with varying ramp rates: $0.25 \mathrm{~kW}, 1 \mathrm{~kW}$ and $4 \mathrm{~kW}$. The other battery parameters are $b_{0}=0.5 \mathrm{kWh}, B_{\text {rated }}=$

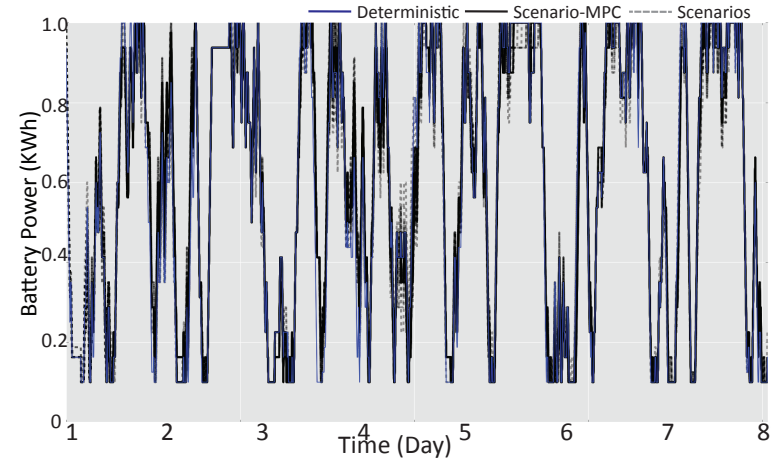

Fig. 5. Visualization of one-week charging/discharging signals under deterministic, single scenario and scenario-MPC control frameworks.

$1 k W h, S o C_{\max }=0.98, S o C_{\min }=0.1, h=\frac{1}{12}$ hour, $\eta_{\mathrm{ch}}=\eta_{\text {dis }}=0.95$. We use one-year locational marginal pricing data from $\mathrm{CAISO}^{4}$ as training data for scenarios, and test the three battery's arbitrage performance on 1-week's separate data. We generate 1,000 independent scenarios at each timestep. This number is selected in our case as we don't observe significant improvement in control performance with more scenarios. During testing on standalone 1-week test data, we also use public available day-ahead forecasts to compare the arbitrage performance.

\section{B. Simulation Results}

We firstly validate if the proposed algorithm can make profits for batteries of varying ramp rates and compare the results with control using full knowledge of price (the deterministic case). As shown in Fig. 4, all three batteries are making profits, but the profits vary a lot depending on the ramp rate. This corresponds to our expectation, since battery with greater ramping rates are able to catch the opportunities of extreme prices (e.g., sell more energy when price are high). We define regret as the ratio of difference on arbitrage gains. The regret comparison is shown in Table I. From Table I we can observe that inaccuracies in uncertainty model affect faster ramping battery (e.g., ultra-capacitor) much more significantly compared to slow ramping battery (e.g., hydro storage). Observe the regret for $4 \mathrm{~kW}$ ramping battery is more

\footnotetext{
${ }^{4}$ http://www.energyonline.com/Data/
} 


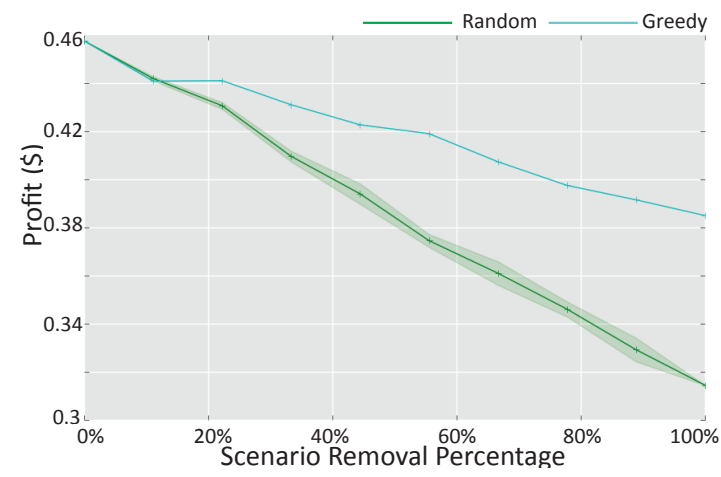

Fig. 6. The impact of scenario removal. We run random removal 10 times with different random seeds during each run.

than $58 \%$ using SMPC, however, for $0.25 \mathrm{~kW}$ ramping battery the regret is less than $3 \%$.

TABLE I

COMPARISON OF REGRET ON ONE-WEEK PROFITS

\begin{tabular}{c|c|c|c}
\hline Max Ramp Rate & Day Ahead & ARIMA & SMPC \\
\hline $0.25 \mathrm{KW}$ & 0.657 & 0.133 & $\mathbf{0 . 0 2 8}$ \\
$1 \mathrm{~kW}$ & 0.855 & 0.237 & $\mathbf{0 . 1 3 4}$ \\
$4 \mathrm{~kW}$ & 0.922 & 0.730 & $\mathbf{0 . 5 8 8}$ \\
\hline
\end{tabular}

In all settings, we observe better performance by using proposed data-driven ScenarioMPC algorithm over MPC using point forecasts (ARIMA) and day-ahead price. More interestingly, when battery ramp rate is lower, ScenarioMPC could achieve nearly the benchmark of using real future price information. When ramp rate is higher, the battery are reacting more strongly to forecast errors, and scenarios can only compensate part of such uncertainties on forecasts.

We then look into the details of how ScenarioMPC affects the battery charging/discharging decisions in Fig. 5, where we show the actions for the battery with $0.25 \mathrm{~kW}$ ramp rate. The decision of each single scenario may deviate from the deterministic version's strategy, while by integrating scenarios into ScenarioMPC, the final decision is getting closer to the deterministic solution. In Fig. 6, we compare the effect of two scenario reduction strategies on final arbitrage, namely random removal and greedy removal, for a battery with $1 \mathrm{~kW}$ ramp rate. In greedy removal algorithm, we remove scenarios with least expected profits. The result suggests that with limited computational resources, greedy scenario removal algorithm will help us select most important scenarios for subsequent control problems without much loss in performance as compared to random removal.

\section{CONCLUSION}

In this paper, we design a novel framework for solving the battery arbitrage problem which is often faced with uncertainties in price forecasts. By utilizing data-driven scenarios in the optimal control problem, simulation results demonstrate our algorithm can achieve better performance than classical MPC algorithms. We will consider a more complex battery model and battery degradation along with scenario removal strategies in our future work.

\section{REFERENCES}

[1] R. Sioshansi, P. Denholm, T. Jenkin, and J. Weiss, "Estimating the value of electricity storage in pjm: Arbitrage and some welfare effects," Energy economics, vol. 31, no. 2, pp. 269-277, 2009.

[2] M. U. Hashmi, A. Mukhopadhyay, A. Busic, and J. Elias, "Optimal control of storage under time varying electricity prices," in IEEE International Conference on Smart Grid Communications, 2017.

[3] K. Bradbury, L. Pratson, and D. Patiño-Echeverri, "Economic viability of energy storage systems based on price arbitrage potential in real-time us electricity markets," Applied Energy, vol. 114, pp. 512-519, 2014.

[4] P. Mokrian, M. Stephen et al., "A stochastic programming framework for the valuation of electricity storage," in 26th USAEE/IAEE North American Conference. Citeseer, 2006, pp. 24-27.

[5] J. Qin, R. Sevlian, D. Varodayan, and R. Rajagopal, "Optimal electric energy storage operation," in Power and Energy Society General Meeting, 2012 IEEE. IEEE, 2012, pp. 1-6.

[6] P. M. van de Ven, N. Hegde, L. Massoulié, and T. Salonidis, "Optimal control of end-user energy storage," IEEE Transactions on Smart Grid, vol. 4, no. 2, pp. 789-797, 2013.

[7] K. Anderson and A. El Gamal, "Co-optimizing the value of storage in energy and regulation service markets," Energy Systems, vol. 8, no. 2, pp. 369-387, 2017.

[8] H. Wang and B. Zhang, "Energy storage arbitrage in real-time markets via reinforcement learning," arXiv preprint arXiv:1711.03127, 2017.

[9] D. Bertsimas, E. Litvinov, X. A. Sun, J. Zhao, and T. Zheng, "Adaptive robust optimization for the security constrained unit commitment problem," IEEE Transactions on Power Systems, vol. 28, no. 1, pp. 52-63, 2013.

[10] J. Schmidli, L. Roald, S. Chatzivasileiadis, and G. Andersson, "Stochastic ac optimal power flow with approximate chance-constraints," in Power and Energy Society General Meeting (PESGM), 2016. IEEE, 2016, pp. 1-5.

[11] M. Campi and G. Calafiore, "Decision making in an uncertain environment: the scenario-based optimization approach," Multiple Participant Decision Making, pp. 99-111, 2004.

[12] K. Abdulla, K. Steer, A. Wirth, S. Halgamuge, and J. de Hoog, "Accounting for forecast uncertainty in the optimized operation of energy storage," in Innovative Smart Grid Technologies-Asia (ISGT-Asia), 2016 IEEE. IEEE, 2016, pp. 183-189.

[13] D. Krishnamurthy, C. Uckun, Z. Zhou, P. R. Thimmapuram, and A. Botterud, "Energy storage arbitrage under day-ahead and real-time price uncertainty," IEEE Transactions on Power Systems, vol. 33, no. 1, pp. 84-93, 2018.

[14] G. Schildbach, L. Fagiano, C. Frei, and M. Morari, "The scenario approach for stochastic model predictive control with bounds on closedloop constraint violations," Automatica, vol. 50, no. 12, pp. 3009-3018, 2014.

[15] Y. Chen, Y. Wang, D. Kirschen, and B. Zhang, "Model-free renewable scenario generation using generative adversarial networks," IEEE Transactions on Power Systems, vol. 33, no. 3, pp. 3265-3275, 2018.

[16] Y. Chen, X. Wang, and B. Zhang, "An unsupervised deep learning approach for scenario forecasts," in 2018 Power Systems Computation Conference (PSCC). IEEE, 2018, pp. 1-7.

[17] M. U. Hashmi, A. Mukhopadhyay, A. Busic, and J. Elias, "Storage optimal control under net metering policies," to be submitted IEEE Transactions on Smart Grid.

[18] M. U. Hashmi and A. Busic, "Limiting energy storage cycles of operation," in Green Technologies Conference (GreenTech), 2018. IEEE, 2018, pp. 71-74.

[19] J. R. Cruise, R. J. Gibbens, and S. Zachary, "Optimal control of storage for arbitrage, with applications to energy systems," in Information Sciences and Systems (CISS), 2014 48th Annual Conference on. IEEE, 2014, pp. 1-6.

[20] A. Parisio, L. Fabietti, M. Molinari, D. Varagnolo, and K. H. Johansson, "Control of hvac systems via scenario-based explicit mpc," in Decision and Control (CDC), 2014 IEEE 53rd Annual Conference on. IEEE, 2014, pp. 5201-5207.

[21] I. Goodfellow, J. Pouget-Abadie, M. Mirza, B. Xu, D. Warde-Farley, S. Ozair, A. Courville, and Y. Bengio, "Generative adversarial nets," in Advances in neural information processing systems, 2014, pp. 2672 2680. 